\title{
Féeries
}

Études sur le conte merveilleux, XVII ${ }^{\mathrm{e}} \mathrm{XIX} \mathrm{X}^{\mathrm{e}}$ siècle

\section{Fables, contes, nouvelles. Liaisons poétiques}

Fable, Tales, Short Stories, Poetic Links

\section{Jean-Paul Sermain}

\section{(2) OpenEdition}

Journals

Édition électronique

URL : http://journals.openedition.org/feeries/752

DOI : $10.4000 /$ feeries. 752

ISSN : 1957-7753

\section{Éditeur}

UGA Éditions/Université Grenoble Alpes

\section{Édition imprimée}

Date de publication : 31 octobre 2010

Pagination : 9-19

ISBN : 978-2-84310-182-3

ISSN : 1766-2842

\section{Référence électronique}

Jean-Paul Sermain, «Fables, contes, nouvelles. Liaisons poétiques », Féeries [En ligne], 7 | 2010, mis en ligne le 31 juillet 2011, consulté le 07 septembre 2020. URL : http://journals.openedition.org/ feeries/752; DOI : https://doi.org/10.4000/feeries.752

\section{(c) Féeries}


Jean-Paul Sermain

Université Paris 3

\title{
FABLES, CONTES, NOUVELLES. LIAISONS POÉTIQUES
}

\author{
À Marc Escola
}

$F$

AUT-IL TRAITER FABLES, CONTES ET NOUVELLES comme des œufs à mettre dans le même panier du récit court? Oui, si l'on entend par ce geste mesurer les relations réciproques par lesquelles chacun se qualifie et se différencie. Mais l'entreprise se révèle compliquée, ou plutôt enrichie, par les changements qui interviennent au fil du temps et que l'on peut mesurer, pour simplifier, en départageant deux grands moments qui coïncident à peu près avec la première modernité (dite Outre-Manche "early modern») et la seconde modernité. Engageons un parcours régressif en commençant par elle. En se basant sur l'usage d'aujourd'hui et la familiarité avec les littératures contemporaines, l'idée de réunir fables et nouvelles nous choque, car contraire à l'impression d'une opposition sinon exclusive du moins tranchée. La nouvelle aujourd'hui s'est considérablement éloignée du conte et de la fable, privilégiant les instantanés, les ellipses, l'expression indirecte qui suggèrent au lecteur ce qu'impliquent un détail, une perception confuse, une rencontre fortuite, des échanges insipides, et laissant donc le récit ouvert, comme une ombre dont la source reste cachée, ou s'interrompant brusquement sans raison apparente. La fable se situe presque aux antipodes : elle est perçue, au moins en France, à partir de l'usage scolaire fait de La Fontaine — jusqu'à récemment, en partie relayé ensuite par le lycée ou l'université et par des transformations médiatiques qui rendent le corbeau et le renard des héros proches, ou encore la cigale et la fourmi. Elle est identifiée à un récit bref, économe dans ses composantes, aux contours particulièrement marqués, et transparent à un sens moral ramassé dans une «moralité» presque toujours énoncée : tout y est subordonné à la formation d'une histoire traitée comme l'exemple d'une signification générale. Loin des pauses, des nuances, des minuties, des dérives et des désinvoltures de la nouvelle actuelle. S'oppose ainsi un genre ancien, que le grand classique résume et 
qu'il laisse sans vraie postérité, désormais associé à l'enfance, aimablement didactique, à un genre moderne pratiqué — certes différemment — par les plus grands écrivains $\mathrm{du} \mathrm{Xx}^{\mathrm{e}}$ siècle.

Le conte se situerait aujourd'hui entre ces deux pôles presque antagonistes et il est moins facile à délimiter. Sa frontière avec la nouvelle, telle qu'elle est reconnue à partir de Pirandello, K. Mansfield, Carver, O'Flaherty, McCullers, Salinger ou Bollaño, tiendrait à la netteté de l'histoire, à son emprise fonctionnelle sur les éléments du texte, à la clarté de son dessein qui permet de le fixer dans la mémoire. Le conte est d'abord une histoire, identifiable, maniable, mémorisable, au point qu'elle se prête à toutes les migrations, appropriations et variations : l'histoire racontée est comme une valeur d'échange. Cette valeur de l'histoire n'exclut pas le sens moral propre à la fable, mais ne l'implique pas non plus et, quand le conte l'accueille, il lui donne une place secondaire et facultative. Un même conte peut servir à des fins persuasives diverses et souvent n'emporte pas l'adhésion sur la leçon qu'il prétend illustrer.

Les choses se compliquent si l'on regarde d'un peu plus près la nouvelle du XIX ${ }^{\mathrm{e}}$ siècle et qu'on considère, par exemple, celles de Maupassant ou de Mérimée : l'opposition au conte et même à la fable n'y est plus si nette. Tout n'y est-il pas soumis à la production d'un effet unique et tendu vers une conclusion qui en ramasse le sens? C'est du moins ce qu'on trouve théorisé chez E. Poe ${ }^{\mathrm{I}}$, comme le remarque Marc Escola ${ }^{2}$ dans un article récent : le tout et son dessein viennent commander dans la nouvelle chaque partie, chaque composante, chaque détail, jusqu'à la mention d'un clou sur le mur. Marc Escola met en rapport cet idéal avec les choix critiques français d'inspiration poétique, virulents dans les années 1960-1970 et analysant le récit selon le principe d'une fonctionnalité régressive : si tout est déterminé par le point d'arrivée, le sens se réduit à une fuite en avant dont le terme est fixé, chaque moment ne valant que par sa relation subordonnée au suivant, la fonction qu'il y remplit — course effrénée inverse mais identique à celle du lecteur qui brûle les étapes pour arriver à la fin qu'il s'est bien gardé de découvrir en premier. Dans son essai sur «la philosophie de la composition», E. Poe insiste sur l'unité de l'effet que doit viser l'œuvre (ce qu'il dit du poème vaut pour la nouvelle), et

I. E. Poe, «La genèse d'un poème», dans Histoires, essais et poèmes, Paris, Le Livre de Poche, coll. "La Pochothèque", 2006, p. I503-1523.

2. M. Escola, "Le Clou de Tchekov. Retours sur le principe de causalité régressive», dans $L a$ Partie et le tout. La composition du roman de l'àge baroque au tournant des Lumières, volume collectif à paraître chez Peeters. 
qui n'est compatible qu'avec une lecture condensée en une fois, donc avec une étendue textuelle faible et un réveil continu de l'intérêt : c'est une sorte de séance voluptueuse. En termes narratifs cela revient à tout subordonner à la fin, comme l'illustre une phrase de Tchekhov rapportée par Tomachevski, que cite Marc Escola :

Pas un seul accessoire ne doit rester inutilisé par la fable. Tchekhov a pensé à la motivation compositionnelle en disant que si au début de la nouvelle on dit qu'il y a un clou dans le mur, à la fin c'est à ce clou que le héros doit se pendre 3 .

L'analyse structurale du récit a privilégié la nouvelle et le conte. Elle a peut-être ainsi renforcé l'illusion trompeuse d'une ressemblance entre les deux récits courts. La nécessité selon Tchekhov ou Maupassant n'a pas la même valeur que dans le conte (d'où la fragilité des analyses formelles). Si nous prenons par exemple La Parure, la nouvelle bien connue de Maupassant, les nombreux détails renvoyant à la réalité matérielle et sociale ne précisent pas seulement circonstances et couleurs pour peindre l'univers représenté mais, conformément aux pratiques romanesques qui s'étendent et s'imposent dans la seconde moitié du XIX ${ }^{\mathrm{e}}$ siècle, ils doivent rendre sensibles les déterminations qui commandent les comportements, les décisions et les sentiments. La réalité n'est plus le langage seulement de l'histoire, elle en devient la règle, faisant du récit le résultat et le miroir des causalités multiples dans lesquelles les individus sont pris. De telle sorte que, si la nouvelle de Maupassant ressemble au conte par la progression des épisodes et la logique qui lui donne sa direction, cette valeur n’est plus rapportée à l'esprit du conteur et du conte (nous allons revenir sur cet aspect autoréflexif du conte) mais à des lois plus générales des sociétés et des psychologies : le sujet même des romans.

Pourtant, hélas!, qui s'arrêterait à ce déterminisme propre au XIX ${ }^{\mathrm{e}}$ siècle risquerait de manquer son usage dans les nouvelles et l'intention des écrivains. Dans La Parure, l'enchaînement des nécessités sociales et individuelles n'a pour horizon ou ultime fin que le sentiment d'une vie gâchée et donc de l'absurde (qu'on trouve dans de nombreuses nouvelles de Tchekhov), d'un tragique sans transcendance (en cela proche de notre époque). Si la nouvelle du XIX ${ }^{\mathrm{e}}$ siècle prend souvent la forme du conte, son foyer sémantique, situé à la fin, est le vide, la négation, la destruction - puisque les personnages sont mus par des appétits de réussite et de plaisirs, comme dans le conte, mais voués à l'échec. Les flammes du récit se réduisent en cendres. La nouvelle, plus proche de nous, aboutit

3. Théorie de la littérature, trad. et éd. de T. Todorov, Paris, Éditions du Seuil, I965, p. 282. 
souvent à ce même effet d'effacement et de vide mais par les moyens tout différents qui nous ont arrêtés d'abord : le déterminisme apparait encore trop lourd et porteur d'un savoir récusé, et s'y substituent le hasard, l'aléatoire, l'accidentel, l'incompréhensible, l'inachevable. Dans la nouvelle de Maupassant, tous les éléments sont rapportés à une même fin, mais cette fin élusive sanctionne l'incapacité à trouver un sens à l'histoire.

Poe, et plus largement les auteurs de nouvelles fantastiques, visent moins à produire un effet de nécessité que sa suspension. La forme de la nouvelle calquée sur celle du conte crée comme une attente d'unité et de sens, vécue par les personnages et adoptée par un lecteur s'aventurant dans les terres familières du conte. Mais c'est finalement pour rendre la réalité et le jugement incertains, pour plonger la conscience dans le trouble. Ce que la nouvelle moderne produit (souvent) par un récit parcellaire et allusif, fait de sensations et de notations triviales, serait obtenu par les romantiques en recourant au modèle du conte et en utilisant son ferme contrôle du récit, sans restes si l'on peut dire, pour arriver à un effet inverse de celui attendu par le conte : ce qui se fixe dans un regard ultime, focalisé et sans distraction, c'est une réalité qui se dérobe, qui se dédouble, qui vacille. Le lien de la nouvelle "fantastique» aux techniques et à l'idéologie réalistes du roman du Xix ${ }^{\mathrm{e}}$ siècle est donc essentiel, en ce que c'est précisément la vision et la confiance en elle propres au roman qui connaissent, dans la nouvelle fantastique, une perturbation : la forme du conte est comme nécessaire à sa déstabilisation. Le Diable amoureux de Cazotte, en ce sens poétique, a enclenché de tels récits (avec les œuvres presque contemporaines de Beckford et de Walpole, mais on pourrait remonter en arrière à une ouvre peu connue de Bibiena, La Poupée) : c'est un conte à la ligne narrative franche et économe dans ses moindres éléments mais qui vise à brouiller le statut même de la femme aimée, de la relation amoureuse et donc de l'histoire en entier — qualifiée d' "espagnole», fortement typée. Le conte comporte une morale mais qui dépend de l'identification de ce qui s'est passé et des personnages : quand ceux-ci deviennent indéterminés, elle reste suspendue à ce brouillard. On a donc là d'emblée une forme mixte, ou peut-être une reprise du conte, pour arriver à une fin contraire d'incertitude et de trouble de la compréhension. Le genre qui donne au récit la forme la plus visible et l'assure du sens le plus évident sert de véhicule au processus de négation et d'inversion; cela explique, comme dans la négation, la présence sous-jacente de l'affirmation comme étape préalable, à la fois formelle et idéologique, comme exigence ou nostalgie d'unité et de simplicité. Maupassant, avec La Parure, met en place un autre détournement du conte qui utilise le principe de l'enchaînement pour faire voir 
le retrait du sens, non plus sa perturbation, mais véritablement sa négation - négation encore rassurante pour nous car elle emprunte la forme close et unifiée du conte et dégage des lois de nécessité pratique, accessibles à la science et peut-être à l'histoire (c'est ce qu'ont cru ses premiers idéologues au début du XIX ${ }^{\mathrm{e}}$ siècle). La nouvelle plus moderne extrait, elle, un fragment de chaos et cherche à en donner l'impression.

Les nouvelles des $\mathrm{XIX}^{\mathrm{e}}$ et $\mathrm{XX}^{\mathrm{e}}$ siècles s'appuient donc sur des formes antérieures du conte. Elles nous invitent ainsi à reculer notre regard sur cette étape préalable : voyons comment ont pu être pratiqués, perçus et disposés les trois genres de la fable, du conte et de la nouvelle dans la période de la "première modernité», "early modern». Cette périodisation elle-même n'est pas satisfaisante, car il faut regarder, pour la fable, l'Antiquité et pour le conte et la nouvelle, le Moyen Âge. Écartons pour l'instant les problèmes de terminologie, car le mot «nouvelle», entre Boccace et $\mathrm{M}^{\mathrm{me}}$ de Lafayette, prend des acceptions bien différentes; il faut au moins se demander si les "nouvelles» de Boccace et de Bandello, voire de Cervantès, et leurs échos français, ne sont pas très proches du conte et si la «nouvelle» du siècle de Louis XIV ne doit pas être vue comme un "petit roman" (ainsi est-elle souvent qualifiée à l'époque). La nouvelle ouvre une zone encore instable, et l'élément le plus ancien et le plus reconnu de notre trilogie est la fable. Sa qualification se fait à la fois par la poétique et par la rhétorique, qui sont alors autant des savoirs que des disciplines d'enseignement. Dans cette seconde perspective, rhétorique, la fable entre au chapitre de l'invention comme un type d'argument et elle est a pour frère l'exemple historique : c'est une sorte d'exemple fictif en forme de récit bref, qui doit soutenir une thèse, ou plus largement une loi générale qui entre dans un raisonnement pour caractériser quelqu'un, l'interprétation d'un événement ou d'une cause. Elle n'est pas une preuve et il va de soi qu'on peut toujours proposer un exemple ou une fable contraire, voire même en tirer des leçons contradictoires. La fable trouve néanmoins sa raison d'être et la logique de sa simplicité et de son unité narrative dans cette idée qu'elle représente ou incarne. Il faut aussi retenir de ce cadre rhétorique que la fable participe à une interprétation (qu'elle soit judiciaire ou politique), qu'elle est donc prise dans un conflit et tiraillée dans des sens opposés.

Le lien de la fable à cet usage oratoire apparaît dans ses représentations, comme celles de La Vie d'Ésope, des romans ou des essais. Surtout, les deux approches rhétorique et poétique de la fable se trouvent associées dans les pratiques pédagogiques nées dans l'Antiquité et revivifiées à partir de la Renaissance. On s'en sert moins pour transmettre une leçon, comme 
dans l'enseignement des III ${ }^{e}$ et $\mathrm{IV}^{\mathrm{e}}$ Républiques, pour servir de support à une «morale» traitée aujourd'hui avec une nostalgie hypocrite quand tout est fait pour "délittérariser» l'enseignement, que pour s'approprier la formation de la leçon : l'élève est invité à intégrer le principe de la fable en en composant, soit à partir d'un noyau narratif, soit à partir d'un énoncé général. Leçon de leçon, si l'on peut dire. La fable entre dans une culture littéraire et le lien entre le récit et la leçon, si évident aujourd'hui, est noué à un autre peut-être plus important entre la fable et son usager, toujours prêt à y recourir ou à l'inventer au besoin d'un discours ou d'un conflit.

Le Moyen Âge ${ }^{4}$ est tout favorable à la fable : dans des recueils d'exemples, au service de l'apologétique chrétienne, de la catéchèse mais aussi de la formation morale, dans la littérature vernaculaire et même dans les échanges avec l'Orient : Kalila et Dimna passe en Europe comme un livre de fables, si l'on peut dire, et se diffuse de façon variée. Dans cette expansion, la fable glisse vers le conte. Le conte est comme une fable qui a mal tourné, c'est-à-dire qui s'affranchit de son ancrage rhétorique et pédagogique (dans le double sens qu'elle fait l'objet d'un apprentissage et qu'elle transmet des leçons utiles). Le récit y garde sa netteté, son économie : tous les éléments narratifs sont subordonnés à la formation et à la perception de l'histoire, et l'affranchissement du rhétorique et du pédagogique conduit à intensifier et à exploiter les qualités propres du récit - complexité, surprise, effet comique ou pathétique, extraordinaire. Le conte dessine un parcours et c'est la valeur de ce parcours qui est décisive. Toutefois, la proximité du conte avec la fable ne tient pas seulement à cette attention au parcours de l'histoire et aux conditions de sa formation; comme la fable, le conte se définit par une intégration au propos et à la personne du "narrateur» (pour reprendre une expression de Boccace ou de Bandello). Le conte, pas plus que la fable, n'est seul : il est associé aux situations de sa production (orale ou écrite), il s'échange, entre dans une mémoire pour se prêter sans cesse à de nouvelles actualisations qui le répandent et le transforment. Mais, alors que l'horizon de la fable est herméneutique - elle participe à un conflit des interprétations - le conte manifeste lui l'esprit du conteur et possède son propre esprit : l'esprit du conteur lui garantit une copia toujours disponible, il consiste surtout à saisir dans une histoire l'organisation qui la rend visible et peut en faire l'agrément, le plus souvent comique, parfois pathétique, toujours surprenant. L'esprit du conte

4. L'Antiquité connaît le conte. Les Métamorphoses d'Apulée assemblent «narrationes et fabulas", indépendantes et dépourvues de toute intention rhétorique ou morale : elles surprennent et amusent. 
est cette qualité que prend le récit, qui séduit et en maintient le souvenir. Le conte ne se définit pas tant par cette rigueur narrative que saisissent les études fonctionnalistes, c'est plutôt un préalable à sa qualification, échappant, elle, à toute description objective.

Le terme et le sens du mot «nouvelle» ont été donnés par Le Décaméron (et nous retiendrons de ces innombrables successeurs les livres copieux de Bandello). Le conte devient une "nouvelle» dans la mesure où elle a pour héros des personnages bien connus, ou dont on a entendu parler ou bien des aventures célèbres, ou méritant de le devenir : les noms, les lieux, les temps dessinent le cadre de la «nouvelle». Le recueil de nouvelles (Boccace, Bandello, Marguerite de Navarre) particularise l'espace virtuel dans lequel se déploient le conte et son esprit : il sélectionne dans la mémoire commune ce qui se prête à devenir une "histoire» avec la couleur du conte. La plupart des contes de Boccace ou de Bandello ne peuvent prétendre à aucune moralité (sinon d'élémentaire prudence ou modération). Pourtant, de nombreuses histoires reposent sur la capacité des personnages à persuader et donc à justifier leurs propres actions : soit par exemple qu'un jeune homme détourne celle qu'il convoite des scrupules religieux en attaquant le clergé, soit, autre exemple, qu'une fille dont le père a tué l'amant revendique sa liberté amoureuse et charnelles. Dans la fable, une telle rhétorique intérieure occupe une place centrale, du moins si nous gardons à l'esprit celles connues de tous de La Fontaine (qui résume pour nous le genre) : pensons au corbeau et au renard, à la cigale et à la fourmi, au loup et à l'agneau, au meunier, son fils et à l'âne, au rat des villes et au rat des champs, au loup et au chien. Dans tous les cas, les personnages sont mis en position rhétorique, chargés de défendre leur point de vue et de fléchir leur adversaire ou leur proie. Les personnages ne résument pas nécessairement la leçon de la fable, bien au contraire, mais celle-ci repose sur l'échange de leurs discours : elle consiste à prendre position dans une configuration rhétorique, à en connaitre les termes et à choisir son camp, ou du moins à savoir qu'il faut choisir dans des relations de conflit oratoire. Chaque position est donc définie de façon rhétorique par un argumentaire, et sa direction est celle de son défenseur : la fable a pour relais de sa leçon, et donc de son usage, le déploiement d'un dialogue rhétorique au sein de l'histoire, plus exactement la fable s'organise sur ce dialogue, y trouve sa dynamique et les éléments de son sens. Avec le conte de Boccace ou Bandello, cette prégnance rhétorique cesse d'être

5. Allusion respectivement à la nouvelle 7 de la troisième journée et à la première nouvelle de la quatrième journée du Décaméron. 
un trait essentiel et devient facultative : elle n'apparaît que dans certaines histoires et contribue plutôt à souligner les contradictions insolubles dans lesquelles se débattent des personnages voués au pire.

Si la nouvelle partage avec le conte son opposition à la fable, elle se distingue de lui par un ancrage explicite dans une situation concrète - aussi bien dans le déroulement de l'histoire que dans sa transmission. Pour autant, la nouvelle de la première modernité annonce-t-elle ses développements de la seconde modernité? La terminologie, très fluctuante, n'est pas de grand secours. Prenons plutôt deux exemples en choisissant des histoires également étendues, complexes, soucieuses du détail concret, amies des péripéties, et tentons de saisir, dans leur opposition, comment se détachent du conte les principes nouveaux de la nouvelle. Soit, d'un côté, l'histoire des trois calenders dans Les Mille et Une Nuits - ample et donnant à de nombreuses circonstances sensibles et à des épisodes pittoresques une grande place (et en cela elle se rapproche bien de la nouvelle). Elle procède d'un projet initial (les frères marient leurs enfants encore à naître), dont l'échec prévisible (ils se disputent sur la dot imaginaire) semble abandonner les personnages à leurs destins indépendants. Toutefois, leurs aventures et leurs décisions reviennent finalement à produire, bien qu'obscures et hésitantes, le résultat initialement prévu : tout ce qui se passe, finalement, entre dans le dessein du conte et sa progression parfaitement visible. Cette sorte de fidélité inconsciente, à une fantaisie et à un rêve, contribue à qualifier le conte et à former son esprit : la surnature d'Éros et des génies, la force des sentiments familiaux réparent merveilleusement les défaillances personnelles et les violences des barbares. L'histoire des trois calenders est un conte porté à un haut niveau de raffinement.

Prenons par contraste La Force du sang, une des Nouvelles exemplaires de Cervantès (1613). Le titre est une expression qui pourrait indiquer la morale de la nouvelle; pour autant cette "force du sang" est problématique : elle intéresse uniquement les grands-parents et pas le père, pourtant plus concerné : ayant dispersé sa semence à tout vent, il part insouciant en Italie et ne revient que sur la proposition de mariage, avec une belle femme, faite par ses parents. C'est donc en sautant une génération que serait effective la "force du sang", mais c'est plutôt le sang versé par l'enfant dans un accident qui suscite la pitié des grands-parents, plus qu'un appel profond de la filiation. Le titre a donc quelque chose d'équivoque et même d'égarant dans la mesure où l'action repose presque toute entière sur la ténacité de l'héroïne violée et parvenant à retrouver le coupable et à l'amener au mariage. Le sang est d'abord celui versé par la victime brutalement déflorée, et il lui donne l'énergie pour élever son enfant et 
amener son bourreau à l'épouser. Comme dans un conte, tous les événements et les moindres circonstances aident à la formation de l'histoire dont le dessein est clair et net : du viol initial au mariage final. Cervantès s'éloigne pourtant du conte et donne ainsi au mot "nouvelle», emprunté des Italiens, une acception inédite et promise à un riche avenir. D'une part la progression de l'histoire ne possède pas de caractère qui pourrait lui servir de fin et d'esprit, elle illustre tout au plus de façon étonnante la force et l'intelligence de l'héroïne. Elle repose sur une série de hasards qui amènent les héros à faire sur le champ des choix décisifs et à chaque fois surprenants : le retour une nuit d'été chez eux de la jeune fille et de ses parents; la rencontre d'un groupe de jeunes gens à chevaux, l'attirance passionnée de l'un d'eux qui revient et enlève la jeune fille qu'il enferme chez lui et viole encore évanouie. Elle le supplie de la libérer et, laissée seule, dérobe un petit crucifix par «un calcul avisé». Ramenée chez elle les yeux bandés, elle ne peut retrouver le lieu de son malheur. Les parents de la jeune fille ont pitié de leur fille et élèvent l'enfant. Celui-ci, assistant à une course de cheval, est renversé et baigne dans son sang. Un gentilhomme, pris de pitié, le prend chez lui pour le faire soigner. Le bruit public attire la mère qui, en le retrouvant, retrouve la pièce où elle a été violée. Le crucifix qu'elle avait dérobé sert de preuve à ceux qui ont recueilli l'enfant et sont ses grands-parents paternels. Elle décide d'obtenir, auprès du crucifix dérobé, ce qu'elle appelle une «réparation». C'est alors qu'elle parvient à convaincre les grands-parents de sa situation, et les amène à faire revenir leur fils et à le marier avec elle.

L'esprit du conte réside dans les coïncidences qui permettent la «réparation» et dans la tension de l'héroïne vers ce but unique. Mais si cette réparation constitue bien le fil directeur du conte, elle n'engage ni les sentiments ni la conscience du père, elle ne pose pas la question de la violence des "appétits» masculins, elle sanctionne et illustre l'éminence d'une héroïne qui échappe à son destin sexuel (le viol) et social (le déshonneur), ou plus exactement qui refuse de voir son identité ramenée à ce tort initialement subi. Au fur et à mesure que l'histoire avance, c'est la liberté des personnages qui est en jeu, et l'attention se fixe sur leur volonté : le choix de l'enlèvement et du viol; le choix de l'héroïne de garder en réserve la mémoire des lieux pour ne pas en rester là; le choix généreux des parents; la pitié devant l'enfant accidenté; la reconnaissance des lieux par l'héroïne; la persuasion des grands-parents; la conception du stratagème pour récupérer un père irresponsable. À chaque moment, la voie prise s'oppose par sa singularité à des comportements normaux, alternativement moraux (ne pas céder à l'appétit) et immoraux (chasser la fille) et elle prend sa 
valeur de cette alternative virtuelle. La succession des actions forme une " histoire exemplaire» dont la ligne nette et belle ressemble à celle d'un conte ou de La Parure de Maupassant, mais les personnages sont laissés toujours libres de leurs décisions et, loin de se mouler dans les déterminismes, ils s'affranchissent de la fatalité.

Cette nouvelle nous donne ainsi le sentiment qu'elle procède du conte, en adopte la logique narrative, l'enchaînement et l'unité, mais tout en exploitant ce qui fait la matière du roman, c'est-à-dire la formation du héros dans des situations ouvertes où il doit apprendre à se conduire audelà des schémas attendus : il se constitue dans une série d'expériences, ici limitées par la brièveté et la concentration que la nouvelle emprunte au conte. Le titre annonce un conte qui résume le parcours d'un rétablissement, d'une réparation, mais Cervantès est sans doute trop catholique et trop romancier pour renoncer à donner plus d'importance à la personnalité de l'héroïne et à son accomplissement : en fondateur de la nouvelle.

De ces exemples on retiendra que le conte peut annexer la fable, s'appuyer sur elle et l'englober dans une logique qui finit pas l'exténuer : alors que la fable repose sur des dispositifs rhétoriques, le conte séduit par l'esprit du récit. Ce que fable et conte ont en commun est qu'ils participent à une culture, c'est-à-dire un espace commun d'échanges, qu'il faut entendre à la fois dans la diffusion des contes et dans la réversibilité des rôles d'auditeur et de producteur. La fable était intégrée à un espace extérieur, celui des discours, celui de la vie (d'Ésope), celui de l'univers politique (dans Kalila et Dimna). La Fontaine marque une rupture en la rapportant au fabuliste : en cela il suit le modèle du conte qui n'a presque de lien qu'avec le conteur. C'est-à-dire que l'auteur même de recueils, de Boccace à Perrault, de Pourrat à Gripari, se place lui-même au sein d'une communauté étendue de conteurs dont il emprunte les matériaux et la posture (ou du moins sa figuration). Cette communauté s'est presque rétrécie aujourd'hui à la jeunesse, à ses formateurs et à ses pourvoyeurs en littérature de divertissement. Ce rétrécissement (qui n’est pas sans restes), cette marginalisation du conte expliquent que l'opposition à la nouvelle se soit faite plus nette. On a vu l'étroite corrélation entre le contenu rhétorique de la fable et son statut rhétorique (soit dans un discours soit par rapport à la moralité). Pour la nouvelle, il faut sans doute, au moins dans sa formation, voir une corrélation similaire entre la liberté des personnages (celle du roman même) et la liberté de l'auteur qui invente et expérimente formes et situations : genre moderne à l'encontre du conte parce qu'il appelait une innovation permanente et la possibilité d'user de la forme brève du récit pour donner à l'achèvement de l'histoire des valeurs inédites 
et concevoir des façons différentes de donner à une situation singulière, à un cas, une portée générale. C'est ce que nous suggèrent les transformations de la nouvelle depuis plusieurs siècles — gardons-nous d'en fixer l'origine! 